\title{
Predicting the spatial organization of chromosomes using epigenetic data
}

\author{
Raphaël Mourad and Olivier Cuvier
}

Please see related articles: http://dx.doi.org/10.1186/s13059-015-0741-y and http://dx.doi.org/10.1186/s13059-015-0740-z

Abstract
Chromosome folding can reinforce the demarcation
between euchromatin and heterochromatin. Two new
studies show how epigenetic data, including DNA
methylation, can accurately predict chromosome folding
in three dimensions. Such computational approaches
reinforce the idea of a linkage between epigenetically
marked chromatin domains and their segregation into
distinct compartments at the megabase scale or
topological domains at a higher resolution.

\section{Introduction}

The ability to probe the spatial organization of chromosomes through the combination of chromosome conformation capture methods with high-throughput sequencing $(3 \mathrm{C}-\mathrm{Hi}-\mathrm{C})$ has revealed how chromosomes organize into active and inactive compartments (indicated 'A' and 'B' , respectively) [1]. This pioneer work highlighted how the fractal organization of chromosomes could favor their folding into individual domains and revealed topologically associating domains (TADs) and sub-TADs (reviewed by Tanay and Cavalli [2]), which represent a pervasive structural feature of the organization of the genome. TADs favor specific long-range contacts between regulatory elements pertaining to the same domain. Such three-dimensional organization of chromosomes into spatially distinct domains sheds light on how complex genomes might set specific transcriptional programs to regulate genes individually or as groups of genes.

Specific long-range contacts formed between enhancers or repressors and their cognate promoters are confined within TADs through additional elements, named insulators or chromatin boundaries, that border
TADs [3-6]. Insulator proteins, including CCCTCbinding factor (CTCF), bind and then recruit the architectural factor cohesin that stabilizes DNA loops formed between distant elements inside TADs, thereby preventing enhancers from targeting ectopic promoters outside of such domains [3-5].

The three-dimensional organization of chromosomes into TADs corresponds with epigenetically defined domains that are marked by specific histone modifications [2]. Such three-dimensional folding of chromosomes participates in the 'setting' of epigenomes, specifically in human cell lineages [7]. Integration of the massive amounts of epigenomic data, including DNA methylation and histone modifications, thus represents a major hurdle for understanding how chromatin organization governs cell identity epigenetically.

\section{Modeling chromosomes in three dimensions}

Although TADs represent a pervasive structural feature of genome organization, approximately one-third of them define more-labile structures that change significantly upon cellular differentiation [7]. Understanding how multiple hierarchical levels of genome organization impact on epigenetic (re-)programming might thus largely rely on integrating $\mathrm{Hi}-\mathrm{C}$ data together with epigenomic data through the development of bona fide computational approaches $[2,8]$. The machine learning technique known as 'random forests' applied to genomic data profiling histone modifications by means of chromatin immunoprecipitation and sequencing (ChIP-Seq) has recently allowed the prediction of $\mathrm{Hi}-\mathrm{C}$ matrices and TAD borders [7]. Such approaches might thus help to unravel the nature and dynamics of epigenomes during cellular differentiation at the molecular level.

* Correspondence: cuvier@ibcg.biotoul.fr

Laboratory of Molecular Biology of Eukaryotes (LBME), CNRS - University of

Toulouse (UPS), F-31000 Toulouse, France 


\section{Epigenome-based prediction of A and B compartments in the nucleus}

Dekker and collaborators originally showed that chromosomes are spatially segregated into three-dimensional compartments $\mathrm{A}$ and $\mathrm{B}$, respectively $[1,8]$. These compartments are cell type-specific, and they strongly associate with euchromatin and heterochromatin, respectively. Two papers take this further. In this issue of Genome Biology, Fortin and Hansen report how they have used such a principle to predict chromosomal compartments from epigenetic domains as defined by DNA methylation data [9]. High methylation in a gene promoter is known to silence the expression of the corresponding gene. Fortin and Hansen successfully predicted A and B compartments in different human cells. For this purpose, they first normalized the Hi-C contact matrix to remove the effect of polymer distance, calculated the correlation matrix and used principal component analysis [9]. The resulting first principal component distinguishes compartments $\mathrm{A}$ and B. Then the authors sought to predict this principal component from methylation data assayed using the available Illumina $450 \mathrm{k}$ microarray platform. Notably, the authors computed a correlation matrix from Illumina $450 \mathrm{k}$ data that highlighted long-range correlations among methylation profiles obtained from different samples. In addition, their methylation-based correlation matrix provided a good prediction accuracy for the $\mathrm{Hi}-\mathrm{C}$ correlation matrix $(R=0.85)$ compared with using the average methylation data profile $(R=0.56)$. A higher accuracy was also obtained $(R=0.93)$ after excluding hard-to-classify genomic loci at the borders of A and B compartments. The authors successfully achieved high prediction accuracy using other epigenetic data such as those resulting from DNase hypersensitivity. Finally, the authors showed the functional implications of such compartment predictions by emphasizing the link with the somatic mutation rate, which is lower in compartment A. Such work is in line with recent data that demonstrated a key role for the three-dimensional organization of chromosomes in setting epigenome landscapes in human cell lineages [7].

\section{Predicting TADs and chromatin interaction hubs}

In a second associated paper in this issue, Huang and colleagues proposed a similar approach to predict TADs by using epigenetic data from various human cell lines, including tumor cells [10]. They used a state-of-the-art computational classifier - Bayesian additive regression trees (BART) - that successfully predicted the presence of TAD borders from the localization of histone modifications or of CTCF insulator protein as inferred from ChIP-Seq data, with a good prediction accuracy (area under the curve $(\mathrm{AUC})=0.77)$. CTCF emerged as the best predictor in the model, in agreement with recent data demonstrating its role in TAD formation $[4,5]$.
In their work, Huang and collaborators also predicted the location of chromatin hubs that play an important role in gene regulation. Chromatin hubs seem to represent complex genomic loci where multiple longrange interactions cluster a number of distant regulatory elements together with the nearby genes. Of note, the authors could show the BART classifier predicted the location of such hubs with high prediction accuracy $(\mathrm{AUC}=0.87)[10]$, which will be of potential significance for unraveling complex genetic disorders.

\section{General implications}

More than 20,000 DNA methylation samples are readily available at Gene Expression Omnibus (GEO) and The Cancer Genome Atlas (TCGA), which might serve to predict three-dimensional chromosome contact maps through approaches similar to those developed by Fortin and Hansen [9]. Computational methods integrating epigenomes and $\mathrm{Hi}-\mathrm{C}$ data clearly represent formidable tools to guide further in-depth analysis of the role of chromosome organization in cell identity [2, 7, 8]. Disease-associated and trait-associated epigenetic variants generated by ENCODE and NIH Roadmap Epigenomics consortia and haplotype-resolved epigenome data have further revealed allele-specific regulatory mechanisms through long-range contact maps during lineage specification [7], which paves the way for understanding the molecular basis of human disease.

Computational approaches contribute to a promising avenue of research in human genetics aiming to unravel key aspects of epigenome regulation through chromosome folding. Fortin and Hansen found long-range correlations among methylation profiles of distant loci, highlighting a coordinated regulation of DNA methylation through three-dimensional clustering of methylated islands. A remaining question is the identity of the molecular drivers of such functional long-range contacts. Our understanding of the regulatory mechanisms of cellular identity, differentiation or reprogramming could thus depend largely on how long-range contacts in chromatin are regulated [7]. Such regulatory events probably involve an interplay between epigenetic regulators and CTCF, cohesin or additional architectural proteins $[3,4,6,7]$.

\section{Concluding remarks}

The papers by Fortin and Hansen and by Huang and colleagues represent successful attempts to predict from epigenetic data higher-order chromatin folding features such as compartments and TADs [9, 10]. Further development of computational approaches using moresophisticated models such as those derived from polymer physics or machine learning should help to improve prediction of $\mathrm{Hi}-\mathrm{C}$ matrices [2,8]. Another major goal is to reconstruct two-dimensional contact maps aiming at 
unraveling the molecular basis of long-range contacts through aggregation of $\mathrm{Hi}-\mathrm{C}$ data [6]. Future models should also integrate epigenomic data together with knowledge of the cognate 'writer', 'reader' and 'eraser' epigenetic factors over the cell cycle. Finally, understanding epigenome propagation might require monitoring the turnover rates of epigenetic marks, which is what conditions 'epigenetic memory', along with the dynamics of long-range contacts.

\section{Abbreviations}

3C-Hi-C: Chromosome conformation capture and high-throughput sequencing; AUC: Area under curve; BART: Bayesian additive regression trees; ChIP-Seq: Chromatin immunoprecipitation and high-throughput sequencing; CTCF: CCCTC-binding factor; TAD: Topological associating domains.

\section{Competing interests}

The authors declare that they have no competing interests.

\section{Authors' contributions}

$\mathrm{RM}$ and $\mathrm{OC}$ jointly wrote this article and have approved the final manuscript.

\section{Acknowledgements}

The authors' research has been supported by grants from the ANR 'INSULa' (OC).

\section{Published online: 29 August 2015}

\section{References}

1. Lieberman-Aiden E, van Berkum NL, Williams L, Imakaev M, Ragoczy T, Telling A, et al. Comprehensive mapping of long-range interactions reveals folding principles of the human genome. Science. 2009;326:289-93.

2. Tanay A, Cavalli G. Chromosomal domains: epigenetic contexts and functional implications of genomic compartmentalization. Curr Opin Genet Dev. 2013;23:197-203.

3. Phillips-Cremins JE, Sauria ME, Sanyal A, Gerasimova TI, Lajoie BR, Bell JS, et al. Architectural protein subclasses shape 3D organization of genomes during lineage commitment. Cell. 2013;153:1281-95.

4. Dowen JM, Fan ZP, Hnisz D, Ren G, Abraham BJ, Zhang LN, et al. Control of cell identity genes occurs in insulated neighborhoods in mammalian chromosomes. Cell. 2014;159:374-87.

5. Rao SS, Huntley MH, Durand NC, Stamenova EK, Bochkov ID, Robinson JT, et al. A 3D map of the human genome at kilobase resolution reveals principles of chromatin looping. Cell. 2014;159:1665-80.

6. Liang J, Lacroix L, Gamot A, Cuddapah S, Queille S, Lhoumaud P, et al. Chromatin immunoprecipitation indirect peaks highlight long-range interactions of insulator proteins and Pol II pausing. Mol Cell. 2014;53:672-81.

7. Dixon JR, Jung I, Selvaraj S, Shen Y, Antosiewicz-Bourget JE, Lee AY, et al. Chromatin architecture reorganization during stem cell differentiation. Nature. 2015:518:331-6.

8. Dekker J, Marti-Renom MA, Mirny LA. Exploring the three-dimensional organization of genomes: interpreting chromatin interaction data. Nat Rev Genet. 2013;14:390-403.

9. Fortin J-P, Hansen KD. Reconstructing A/B compartments as revealed by Hi-C using long-range correlations in epigenetic data. Genome Biol. 2015; doi:10.1186/s13059-015-0741-y.

10. Huang J, Marco E, Pinello L, Yuan G-C. Predicting chromatin organization using histone marks. Genome Biol. 2015; doi:10.1186/s13059-015-0740-z. 